\title{
Terapia de reemplazo renal continua en nińos: de los conceptos a la prescripción
}

\section{Continuous renal replacement therapy in children. From concepts to prescription}

Oscar Camilo Pantoja-Gómez ${ }^{1}$, Mónica Vanessa Mondragón-Gaviria² ${ }^{2}$ Andrea Carolina Lasso-Florez ${ }^{3}$, Rubén Eduardo Lasso-Palomino ${ }^{4}$

RESUMEN

La lesión renal aguda es considerada como una disminución abrupta de la función renal que genera acumulación deproductos de desecho, pérdida de balance de líquidos y electrolitos, y alteración de la homeostasis ácido-base. Es una entidad con alta incidencia que afecta a pacientes pediátricos en estado crítico. Uno de cada 10 pacientes afectados por esta patología requeriná terapia de

\section{ABSTRACT}

Acute kidney injury is considered as an abrupt decrease in kidney function that generates an accumulation of waste products, loss of fluid and electrolyte balance, and alteration of acid-base homeostasis. It is an entity with a high incidence that affects pediatric patients in critical condition. One in 10 patients affected by this pathology will require renal replacement therapy for

\section{Historial del artículo:}

Fecha de recepción: 10/05/2021

Fecha de aprobación: 26/05/2021

1 Universidad del Cauca, Residente de Pediatría, Popayán Colombia.

2 Universidad del Cauca, Pediatra, Popayán, Colombia.

3 Universidad Nacional de Colombia, Enfermera, Bogotá, Colombia.

4 Universidad CES, Pediatra e Intensivista Pediatra, Medellín, Colombia.

Correspondencia: Oscar Camilo Pantoja Gómez. Dirección: Carrera 6 No 13N-50. Facultad de Salud. Universidad del Cauca. Popayán Colombia. Celular: 317668 8415. Correo electrónico: opantojag@unicauca.edu.co

Como citar este artículo: Pantoja-Gómez 0, Mondragón-Gaviria M, Lasso-Florez A, Lasso-Palomino R. Terapia de reemplazo renal continua en nińos: de los conceptos a la prescripción. Revista de la Facultad de Ciencias de la Salud de la Universidad del Cauca. 2021;23(1):32-46. https://doi.org/10.47373/rfcs.2021.v23.1924 
reemplazo renal para su manejo. En la actualidad existe un conjunto de modalidades terapéuticas que permiten la circulación continua de sangre a través de circuitos extracorpóreos con el fin de soportar y/o reemplazar la función normal de los rinones en forma continua durante las 24 horas del día. Tales modalidades se agrupan dentro del término: "Terapia de reemplazo renal continua" (TRRC) y tienen varias ventajas sobre los métodos tradicionales; no obstante, existe entre los profesionales de la salud cierto temor a la hora de prescribir estas terapias debido a su complejidad. En esta revisión de la literatura se presentan los conceptos básicos, modalidades e indicaciones de la TRRC en nińos de forma práctica para facilitar su prescripción.

Palabras clave: Lesión, Renal, Terapia, Reemplazo, Nińo (DeCS)

\section{INTRODUCCIÓN}

Al referirnos a terapia de reemplazo renal continua (TRRC) hablamos de un conjunto de modalidades terapéuticas que incluyen circulación continua de sangre a través de circuitos extracorpóreos, con el fin de soportar y o reemplazar lo mejor posible la función normal de los rińones en forma continua durante las 24 horas del día (1, 2). Estas terapias son especialmente idóneas en pacientes inestables hemodinámicamente, con lesión renal aguda (LRA) y sobrecarga hídrica, condiciones frecuentemente observadas en pacientes que se encuentran en unidades de cuidado intensivo pediátrico (UCIP) y que han permitido disminuir la morbimortalidad y mejorar su calidad de vida (3).

La TRRC ha evolucionado de manera significativa. En 1977, Peter Kramer cambió la hemodiálisis intermitente tradicional por un nuevo tratamiento llamado hemofiltrado continuo arteriovenoso, procedimiento basado en una membrana (filtro) altamente permeable, conectado a una arteria y a una vena mediante accesos vasculares de hemodiálisis modificada, logrando la producción lenta de un ultrafiltrado y la sustitución de líquidos para mantener un adecuado balance. En ańos recientes, la técnica se ha modificado y se han introducido diferentes innovaciones que conforman las actuales modalidades de TRRC $(1,4)$.

Aunque se conocen los beneficios de la TRRC, existe entre los profesionales de la salud cierto temor a la hora its management. Currently, there is a set of therapeutic modalities that allow continuous circulation of blood through extracorporeal circuits to support and/or replace the normal function of the kidneys continuously 24 hours a day. Such modalities are grouped under the term: "Continuous renal replacement therapy" (CRRT) and have several advantages over traditional methods; however, there is some fear among health professionals when prescribing these therapies due to their complexity. In this literature review, the basic concepts, modalities, and indications of CRRT in children are presented in a practical way to facilitate its prescription.

Keywords: Acute Kidney Injury, Continuous Renal Replacement Therapy, Child

de prescribir la terapia debido a su complejidad. Por esta razón, el objetivo de esta revisión es presentar los conceptos básicos, modalidades e indicaciones de la TRRC de forma práctica para facilitar su prescripción.

\section{CONCEPTOS BÁSICOS}

\section{LESIÓN RENAL AGUDA}

Se define como una disminución abrupta de la capacidad renal para llevar a cabo sus funciones; se caracteriza por la acumulación de productos de desecho, pérdida de balance de líquidos y electrolitos, y alteración de la homeostasis ácido-base. Es una entidad frecuente en pacientes en estado crítico, que puede ser o no reversible $(5,6)$. Aunque existen múltiples causas de Lesión renal aguda (LRA), estas varían de acuerdo a las características clínicas y demográficas de cada paciente, comorbilidades como sepsis, hipotensión, deshidratación y tratamientos como ventilación mecánica, terapia vasoactiva, trasplantes de órganos y cirugía cardiovascular $(4,6,7)$.

La incidencia de LRA a nivel global se reporta entre el 26 y $32 \%$. En Colombia, de acuerdo con estudios realizados en instituciones de alta complejidad, la incidencia se encuentra entre el 5 y $11,5 \%$. Se describe que hasta un $10 \%$ de los pacientes con LRA requerirán terapia de reemplazo renal (TRR) y corresponde a un factor de riesgo 
independiente para mortalidad, la cual se reporta entre el 30 y $50 \%(5,8-10)$.

La definición de LRA ha cambiado a través de los ańos, debido al uso de diferentes clasificaciones como pRIFLE y AKIN. Actualmente su definición se establece siguiendo las recomendaciones de la Disease Improving Global Outcomes (KDIGO), que diagnostica LRA cuando el paciente tiene un incremento en la creatinina sérica $\geq 0.3 \mathrm{mg} / \mathrm{dl}$ en 48 horas $0 \geq 1.5$ veces la creatinina basal en un periodo de 7 días, 0 una disminución en el gasto urinario $<0,5 \mathrm{ml} / \mathrm{kg} / \mathrm{h}$ durante 6 horas. La clasificación de la LRA, según KDIGO, se resume en la Tabla $1(11,12)$.

Tabla 1. Clasificación KDIGO de LRA.

\begin{tabular}{|c|c|c|}
\hline & $\begin{array}{l}\text { Creatinina } \\
\text { Sérica (CrS) }\end{array}$ & Diuresis \\
\hline Estadio 1 & $\begin{array}{l}\text { Aumento de CrS } \\
\geq 0.3 \mathrm{mg} / \mathrm{dl} \mathrm{o} \\
\text { aumento } 1.5 \mathrm{a} \\
1.9 \text { veces el valor } \\
\text { basal. }\end{array}$ & $\begin{array}{l}<0.5 \mathrm{ml} / \mathrm{kg} / \mathrm{h} \text { durante } \\
6 \text { a } 12 \text { horas. }\end{array}$ \\
\hline Estadio 2 & $\begin{array}{l}\text { Aumento } \geq 2 \mathrm{a} \\
2.9 \text { veces el valor } \\
\text { basal. }\end{array}$ & $\begin{array}{l}<0.5 \mathrm{ml} / \mathrm{kg} / \mathrm{h} \text { por más } \\
\text { de } 12 \text { horas. }\end{array}$ \\
\hline Estadio 3 & $\begin{array}{l}\text { Aumento } \geq 3 \text { ve- } \\
\text { ces el valor basal } \\
\text { o } \mathrm{CrS} \geq 4 \mathrm{mg} / \mathrm{dl} \text { o } \\
\text { inicio de TRR }\end{array}$ & $\begin{array}{l}<0.3 \mathrm{ml} / \mathrm{kg} / \mathrm{h} \text { por más } \\
\text { de } 24 \text { horas o anuria } \\
\text { por más de } 12 \text { horas. }\end{array}$ \\
\hline
\end{tabular}

El tratamiento de la LRA varía desde la protección renal hasta la terapia de soporte y o reemplazo renal.

\section{TERAPIA DE REEMPLAZO RENAL CONTINUA}

Es un término general que se refiere a cualquier terapia extracorpórea que logra la depuración de sustancias mediante membranas semipermeables y logra sustituir la función renal durante las 24 horas del día. Tiene como objetivo principal la remoción de solutos y líquidos del compartimiento intravascular de manera lenta y continua, sin incrementar el riesgo de inestabilidad hemodinámica, disminuyendo así el riesgo de isquemia secundaria.

La terapia de reemplazo renal continua presenta ventajas relacionadas con la prevención y manejo de la sobrecarga de líquidos y la regulación del equilibrio electrolítico y ácido base. Esto permite el uso de terapias de apoyo como transfusiones de hemoderivados, nutrición parenteral, inmunomodulación en sepsis, entre otras. Es recomendada en escenarios de LRA en trauma craneoencefálico, debido a que produce menos fluctuaciones en la osmolaridad plasmática, el volumen, y favorece su recuperación; se realiza con modificaciones mínimas de la osmolaridad plasmática y del volumen intravascular, sin alteración dramática de la hemodinamia (1-3,13).

Para entender los mecanismos desarrollados en las modalidades de TRRC, es necesario recordar los siguientes principios:

\section{Ósmosis}

Fenómeno físico-químico que hace referencia al paso del agua de un sitio de menor a uno de mayor concentración de solutos, a través de una membrana permeable, hasta alcanzar un equilibrio de concentración y presión.

\section{Difusión Simple}

Proceso por el cual los solutos pasan a través de una membrana permeable de un área de mayor concentración a una de menor concentración, mediante movimientos moleculares aleatorios, hasta lograr el equilibrio de los solutos. La transición de solutos dependerá del gradiente de concentración, superficie de la membrana y su permeabilidad. Mediante difusión se eliminan moléculas de bajo peso molecular de modo efectivo.

\section{Convección}

Movimiento en masa de moléculas a través de una membrana semipermeable de alto flujo. En TRRC consiste en la remoción de solutos mediante su "arrastre" en el ultrafiltrado a través de una membrana de alta permeabilidad. El volumen de agua plasmática ultrafiltrada se repone simultáneamente con una solución especialmente desarrollada para tal fin (líquido de reposición o sustitución); los solutos eliminados mediante convección incluyen peso molecular bajo y medio.

Los principios de difusión y convección se muestran en la Figura 1. 
Figura 1. Principios de difusión y convección.
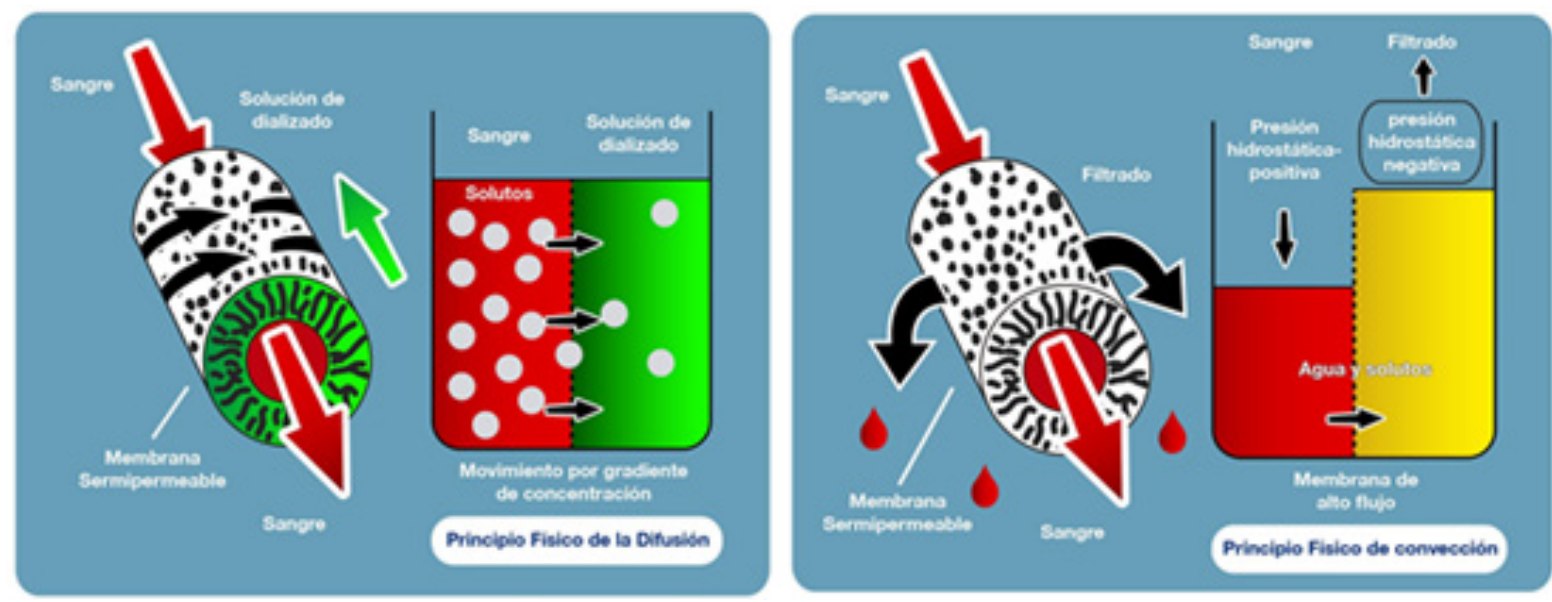

Adaptado de: Poveda R, Fajardo C, et al. Terapia de reemplazo renal continua en el paciente con oxigenación por membrana extracorpórea: consideraciones para la combinación de ambas terapias. Rev. méd. Chile. 2018;146(1):78-90.

\section{Presión osmótica}

Fuerza necesaria que, al ser aplicada, evita el flujo del solvente del lado de menor concentración de solutos al de mayor concentración; en caso del agua se llama presión oncótica.

\section{Ultrafiltración}

Movimiento de líquidos a través de una membrana semipermeable, impulsados por un gradiente de presión; algunos solutos de bajo peso molecular pueden acompańar el agua plasmática ultrafiltrada dependiendo de su tamańo molecular.

\section{Adsorción}

Es la adhesión selectiva de moléculas a la pared de la membrana del filtro, de acuerdo a propiedades físico químicas desarrolladas específicamente para lograr dicho efecto.

Se lleva a cabo mediante la unión selectiva y no selectiva de moléculas a la propia membrana del filtro, y contribuye al aclaramiento de moléculas de tamańo medio y grande.

Adicionalmente, existen términos relacionados con la prescripción que se deben recordar. Estos son:
Filtros: son membranas semipermeables con capacidad para remover solutos durante la TRRC.

Predilución: administración de líquido de reemplazo y terapéutico a la sangre del paciente antes del filtro.

Postdilución: administración de líquido de reemplazo y terapéutico a la sangre del paciente posterior al hemofiltro.

Líquido de tratamiento: solución farmacéutica especializada, fabricada para las TRRC; contiene electrolitos y amortiguadores buffer que sustituyen las pérdidas del agua y electrolitos durante la hemofiltración o hemodiafiltración. Su función es bimodal: difusión y convección, por lo que puede ser utilizado como dializante o como líquido de reemplazo.

Dializante: solución farmacéutica especializada fabricada para las CRRT, que provoca un gradiente de concentración a través del filtro, facilitando la difusión de solutos.

Efluente: se refiere al fluido producido como resultado de desarrollar los mecanismos de ultrafiltración, difusión y convección; dicho líquido puede ser agua ultrafiltrada proveniente del paciente (ultrafiltrado), dializante o una combinación de ambas según la modalidad elegida (14).

\section{INDICACIONES DE LAS TRRC}

La indicación de estas terapias será realizada de forma individualizada por los profesionales tratantes en cada UCIP y debe prescribirse ante una situación clínica que 
requiera del soporte o reemplazo de la función renal u otra condición clínica corregible con estas terapias. Se realiza teniendo en cuenta factores como la severidad de la LRA, el estado clínico del paciente y las siguientes indicaciones (1-4,15,16):

- Insuficiencia cardíaca congestiva y cirugía cardíaca en aquellos pacientes que no mejoran con el tratamiento convencional.

- Falla hepática, específicamente en encefalopatía hepática.

- Intoxicaciones por medicamentos como: procainamida, litio, salicilatos, medio de contraste u otros compuestos dializables.

- Tratamiento de acidosis láctica y metabólica (con $\mathrm{pH}<7.0$ ), a través de la administración de grandes cantidades de bicarbonato, sin riesgo de producir hipernatremia ni sobrecarga de fluidos que se logra mediante la TRRC.

- Alteraciones electrolíticas, como hiperkalemia e hipernatremia severa. También se puede realizar la infusión de electrolitos en los líquidos de reposición en caso de que los niveles se encuentren bajos.

- Hipertermia e hipotermia graves y refractarias al tratamiento convencional, a través del enfriamiento o calentamiento de la sangre en los circuitos extracorpóreos del paciente.

- Grandes quemados, la TRRC permite un control óptimo del estado catabólico y del manejo de fluidos, con disminución de complicaciones.

- Oliguria o anuria persistentes

- Rabdomiólisis en pacientes con lesiones de aplastamiento, para prevenir complicaciones.

- Uremia sintomática

- En la falla multiorgánica, la TRRC mejora el flujo sanguíneo, con una mejor redistribución a nivel periférico, hasta las células previamente hipóxicas, obteniéndose un descenso relativo de la mortalidad.
- Sobrecarga de volumen, cuyo porcentaje se calcula así:

\% de sobrecarga = Líquidos administrados - Líquidos eliminados x 100

Peso de admisión a UCIP

Los estudios indican que la mortalidad incrementa si la sobrecarga es $>10-15 \%$, por lo que este valor debe ser considerado para el inicio de la TRRC.

Se prefiere el inicio de TRRC de forma temprana, sin embargo, depende de la disponibilidad tecnológica en cada UCIP; no hay que olvidar que las modalidades de TRRC ofrecen la posibilidad de mantener la eliminación de volumen, personalizar los líquidos de sustitución de acuerdo con el estado clínico del paciente y combinar los métodos de depuración, manteniendo mayor estabilidad hemodinámica por efectos clínicos y bioquímicos $(17,18)$.

\section{MODALIDADES DE TRRC}

La TRRC tiene cuatro modalidades: ultrafiltración lenta continua, hemofiltración venovenosa continua, hemodiálisis venovenosa continua y hemodiafiltración venovenosa continua; en todas las modalidades se utiliza un circuito extracorpóreo originado en una vena central, y que termina en una vía central. Cada una de las modalidades tiene diferentes principios e indicaciones específicas que se describen a continuación (1-3):

\section{Ultrafiltración lenta continua}

También se conoce como SCUF. Esta modalidad utiliza el principio de ultrafiltración, por medio del cual se remueve la sobrecarga hídrica a través de la extracción de ultrafiltrado calculado y programado de forma lenta desde la circulación sanguínea a través del filtro, sin ningún tipo de reposición o diálisis. No hay líquido de sustitución ni reemplazo, porque el objetivo primordial es retirar el exceso de volumen del paciente. Por lo tanto, es útil en casos de sobrecarga hídrica con función renal adecuada, pero con inadecuada respuesta a diuréticos, como se explica en la Figura 2. 
Figura 2. Ultrafiltración lenta continua.

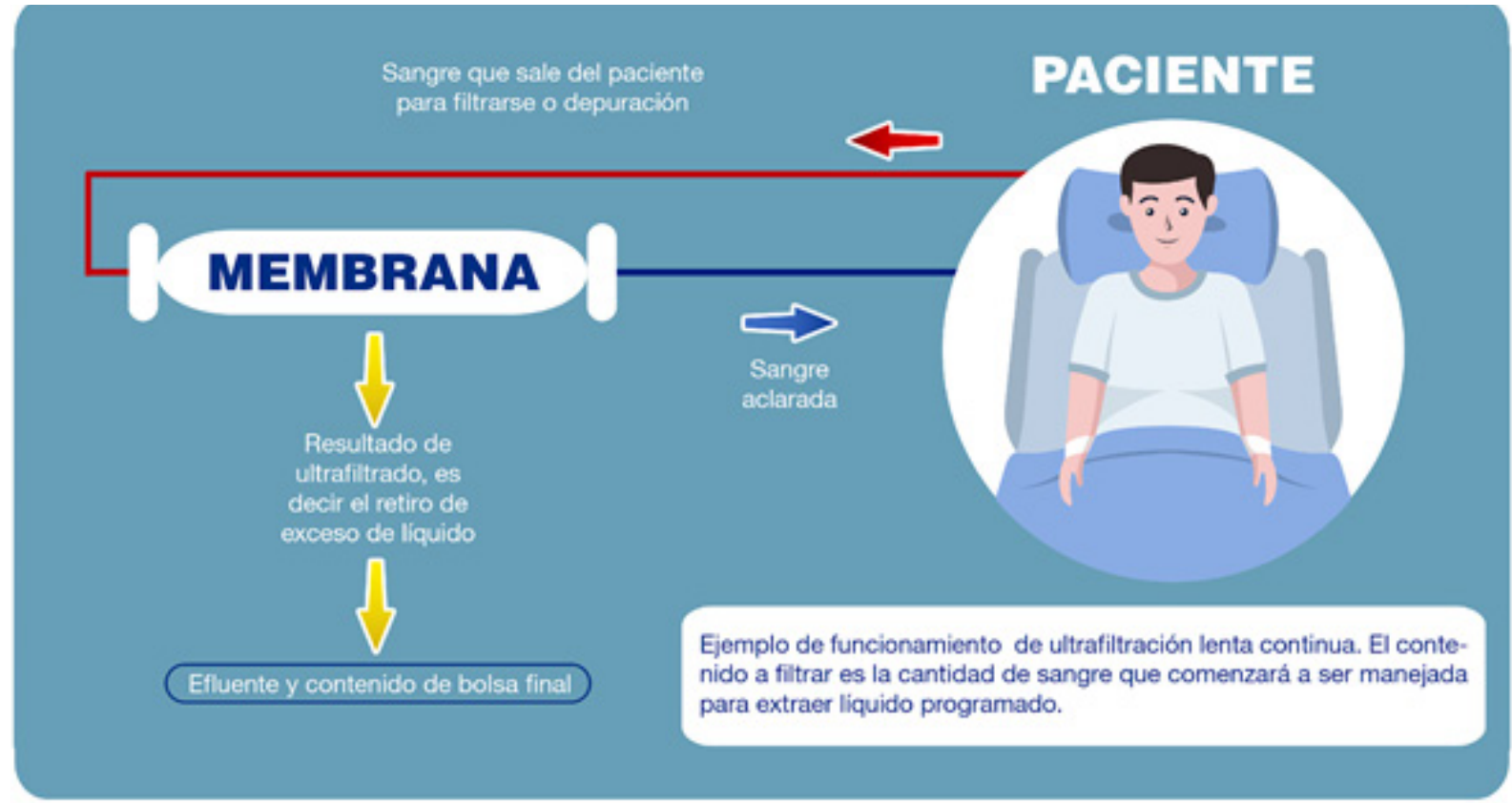

Adaptado de: Sosa-Medellín MÁ, Luviano-García JA. Continuous renal replacement therapy. Concepts, indications and basic aspects of its program. Med Interna Mex. 2018;34(2):288-98.

\section{Hemofiltración venovenosa continua}

Permite extracción de agua ultrafiltrada desde la circulación sanguínea y depuración de solutos mediante convección. En términos prácticos, esta modalidad consiste en hacer pasar el flujo de sangre a través de un filtro de alta permeabilidad hidráulica (se elimina agua y solutos a través de un filtro al ejercer un gradiente de presión positiva y negativa). El ultrafiltrado es mayor a las pérdidas del paciente, por tanto, se necesitará líquido de reemplazo. Se indica en el manejo de exceso de líquidos, sepsis, pacientes quemados, rabdomiolisis y eliminación de moléculas de tamańo mediano. Figura 3.

\section{Hemodiálisis venovenosa continua.}

En esta modalidad se usa la difusión. Consiste en hacer pasar un flujo programable del dializante a contracorriente del flujo sanguíneo, consiguiendo asíla eliminación por difusión de moléculas de pequeńo tamańo. A medida que el dializante pasa a través de la cámara externa del filtro, las moléculas a las cuales la membrana es permeable se mueven del plasma a la solución dializante, la cual finalmente es desechada. Es decir, permite extracción de agua ultrafiltrada desde la circulación sanguínea y depuración de solutos mediante difusión a través del filtro; sus indicaciones principales son uremia y desequilibrios electrolíticos. Figura 4. 
Figura 3. Hemofiltración venovenosa continua.

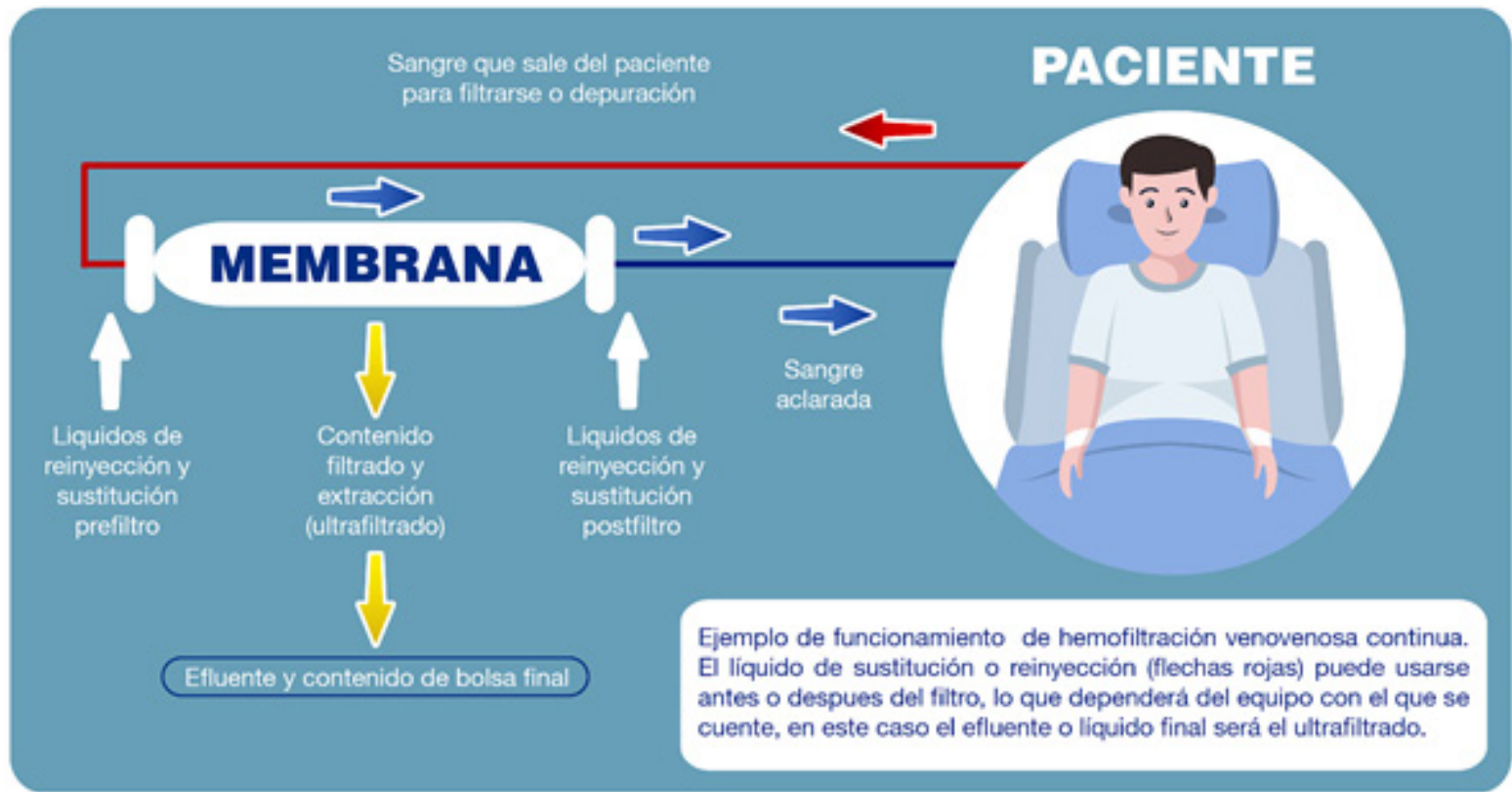

Adaptado de: Sosa-Medellín MÁ, Luviano-García JA. Continuous renal replacement therapy. Concepts, indications and basic aspects of its program. Med Interna Mex. 2018;34(2):288-98.

Figura 4. Hemodiálisis venovenosa continua.

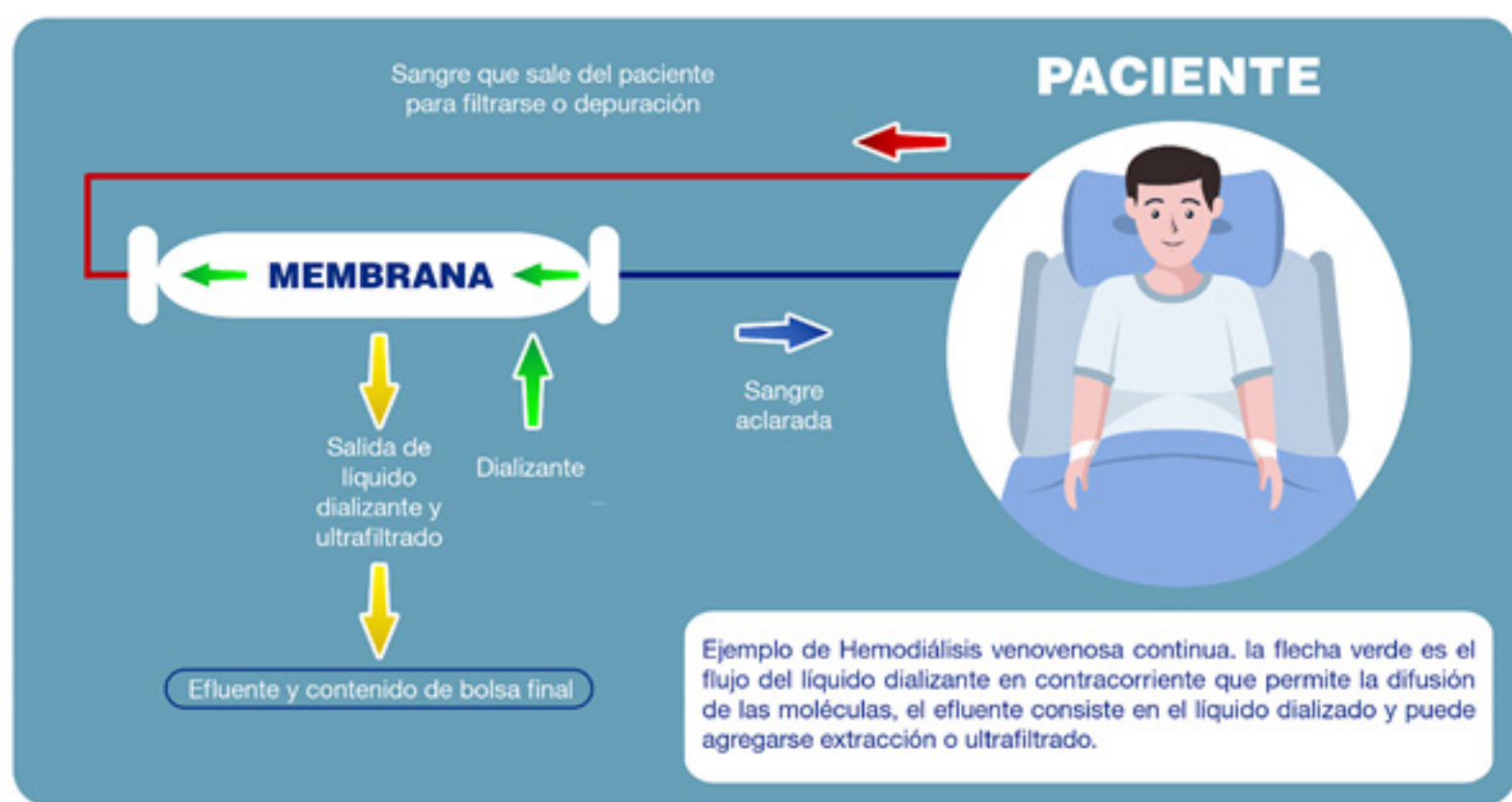

Adaptado de: Sosa-Medellín MÁ, Luviano-García JA. Continuous renal replacement therapy. Concepts, indications and basic aspects of its program. Med Interna Mex. 2018;34(2):288-98. 


\section{Hemodiafiltración venovenosa continua}

Esta modalidad combina los mecanismos de hemodiálisis venovenosa continua y la hemofiltración venovenosa continua, es decir, se utilizan los principios de convección y difusión. Con esta modalidad se puede remover moléculas de mediano y bajo peso; por ejemplo, puede ser útil en el caso de LRA inducida por trauma (rabdomiólisis). Otras indicaciones adicionales son sepsis y falla orgánica múltiple. Al utilizar esta modalidad es necesaria la reinyección de una solución especializada para TRRC, que puede ser prefiltro y/o postfiltro, de acuerdo con la posibilidad técnica de la tecnología con la que se cuente, la cual puede conseguir el adecuado equilibrio de fluidos. Figura 5.

La Tabla 2 muestra un comparativo entre las modalidades de TRRC descritas anteriormente:

Figura 5. Hemodiafiltración venovenosa continua.

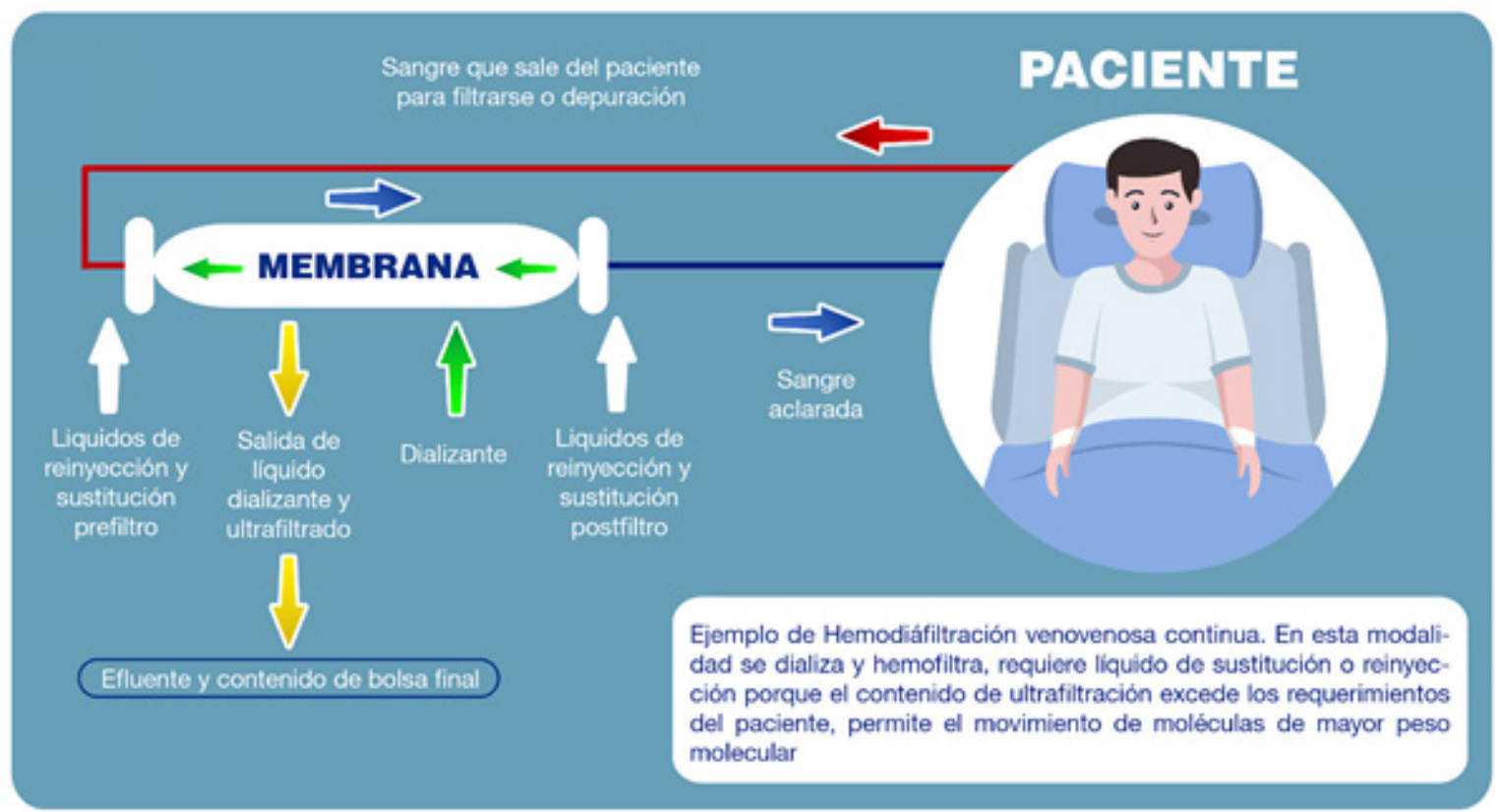

Adaptado de: Sosa-Medellín MÁ, Luviano-García JA. Continuous renal replacement therapy. Concepts, indications and basic aspects of its program. Med Interna Mex. 2018;34(2):288-98.

Tabla 2. Comparación de las modalidades de TRRC

\begin{tabular}{lllll}
\hline & \multicolumn{1}{c}{$\begin{array}{c}\text { Ultrafiltración lenta } \\
\text { continua. }\end{array}$} & $\begin{array}{c}\text { Hemofiltración ve- } \\
\text { novenosa continua. }\end{array}$ & $\begin{array}{c}\text { Hemodiálisis veno- } \\
\text { venosa continua. }\end{array}$ & $\begin{array}{c}\text { Hemodiafiltración } \\
\text { venovenosa continua. }\end{array}$ \\
\hline $\begin{array}{l}\text { Mecanismo } \\
\begin{array}{l}\text { Tiempo de trata- } \\
\text { miento }\end{array}\end{array}$ & Continuo & Difusiltración & Consión y convección & Continuo \\
$\begin{array}{l}\text { Solución de trata- } \\
\text { miento }\end{array}$ & No & $30-4000 \mathrm{ml} / \mathrm{h}$ & No & $70-4000 \mathrm{ml} / \mathrm{h}$ \\
& Heparina, citrato, & $\begin{array}{l}\text { Heparina, citrato, } \\
\text { ninguna }\end{array}$ & $\begin{array}{l}\text { Heparina citrato, } \\
\text { ninguna }\end{array}$ & Heparina, citrato, ninguna \\
\hline
\end{tabular}




\section{RECOMENDACIONES PARA LA PRESCRIPCIÓN}

Con el objetivo de realizar una intervención temprana en los pacientes que estén en riesgo de desarrollar LRA, se sugiere la realización del índice de angina renal (IAR) en las primeras 12 horas a partir del ingreso a la UCIP.
Esto permite determinar de manera temprana qué grupo de pacientes se pueden beneficiar del uso de diuréticos, estrategias dirigidas de nefroprotección y TRRC. El IAR se calcula como se explica en la Tabla 3:

Tabla 3. Índice de Angina Renal.

\begin{tabular}{|c|c|c|}
\hline Índice de Angina Renal & & Puntaje \\
\hline \multicolumn{3}{|l|}{ Riesgo de LRA } \\
\hline $\begin{array}{l}\text { Moderado: } \\
\text { Ingreso a UCIP }\end{array}$ & & 1 \\
\hline $\begin{array}{l}\text { Alto: Trasplante renal o de } \\
\text { médula ósea }\end{array}$ & & 3 \\
\hline $\begin{array}{l}\text { Muy alto: Pacientes en ventila- } \\
\text { ción mecánica invasiva o uso de } \\
\text { inotrópicos o vasopresores }\end{array}$ & & 5 \\
\hline \multicolumn{3}{|l|}{ Evidencia de LRA } \\
\hline Disminución de la TFG & \% de sobrecarga hídrica & \\
\hline Sin cambios & $<5 \%$ & 1 \\
\hline $0-25 \%$ & $\geq 5-10 \%$ & 2 \\
\hline $25-50 \%$ & $\geq 10$ & 4 \\
\hline$>50 \%$ & $\geq 15$ & 8 \\
\hline
\end{tabular}

Se sugiere que un puntaje en el IAR igual o superior a 8 puntos asociado a una elevación $>20$ del biomarcador NGAL (lipocalina asociada con la gelatinasa de neutrófilos), considerado como un biomarcador urinario que predice el desarrollo y evolución de la LRA, sea indicación de realizar un reto diurético con furosemida como intervención temprana. En caso de no contar con este biomarcador, realizar el reto diurético ante la instauración de la LRA a partir del estadio KDIGO 1 (19).
Si el puntaje es menor a 8 puntos, se recomienda realizar estrategias de protección a través del manejo de líquidos endovenosos, medidas que aseguren la perfusión y disminuir o ajustar el uso de nefrotóxicos. En la Figura 6 se indica el algoritmo propuesto. 
Figura 6. Algoritmo de intervención temprana basado en IAR

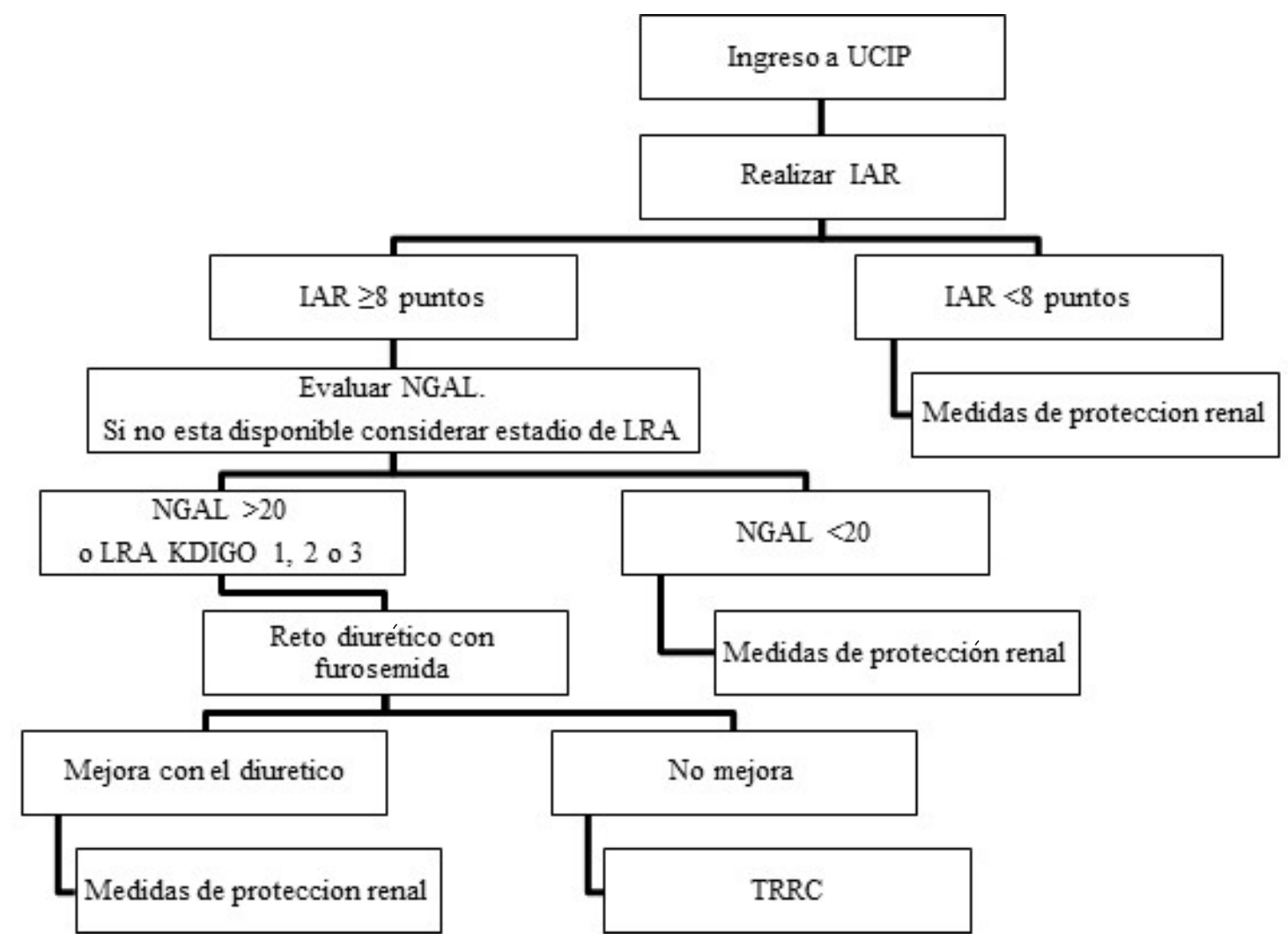

Elaborado por: Pantoja G, Mondragón M, Lasso A y Lasso R.

Abreviaturas: LRA, Lesión Renal Aguda; UCIP, Unidad de cuidado intensivo pediátrico; TFG, Tasa de filtración glomerular; TRRC, Terapia de reemplazo renal continua.

En caso de no evidenciarse mejoría tras el "reto renal", o de presentarse deterioro de la función renal o aumento de la sobrecarga hídrica, se sugiere el inicio de TRRC. Se recomienda tener en cuenta los siguientes parámetros:

\section{Acceso vascular}

Es el aspecto más importante, ya que, al utilizar un acceso vascular correcto, se permite una terapia adecuada con una mejor tasa de aclaramiento sanguíneo y se previene la pérdida accidental o no voluntaria de sangre. En los escenarios donde el flujo es inadecuado, se corre el riesgo de que se coagule el circuito extracorpóreo (20). En la Tabla 4 se indica el tamańo del catéter a utilizar según el tamańo o el peso del paciente.
Tabla 4. Tamańo del catéter para accesos vasculares

\begin{tabular}{rc}
\hline Tamańo paciente & Tamańo catéter \\
\hline Neonatos & $7 \mathrm{Fr}$ \\
$3-6 \mathrm{~kg}$ & $7 \mathrm{Fr}$ \\
$6-12 \mathrm{~kg}$ & $8 \mathrm{Fr}$ \\
$12-20 \mathrm{~kg}$ & $9 \mathrm{Fr}$ \\
$20-30 \mathrm{~kg}$ & $10 \mathrm{Fr}$ \\
$>30 \mathrm{~kg}$ & $10-12 \mathrm{Fr}$ \\
\hline
\end{tabular}

Una vez se elige el catéter, debemos seleccionar la vena para la inserción de este. Las opciones disponibles son: 
- Primera opción: vena yugular derecha

- Segunda opción: venas femorales

- Tercera opción: vena yugular izquierda

- Última opción: vena subclavia con preferencia del lado dominante

\section{Ritmo flujo sanguíneo}

El ritmo de flujo sanguíneo depende del catéter disponible, en general el flujo promedio es de 5-12 $\mathrm{ml} / \mathrm{kg} / \mathrm{min}$, el ritmo minuto es $30-150 \mathrm{ml} / \mathrm{min}$ (para disminuir coagulación en el acceso vascular y el filtro) y el ritmo máximo es $300 \mathrm{ml} / \mathrm{min}$. Este ritmo debe permitir una presión de entrada menor a $-150 \mathrm{mmHg}$, las tecnologías disponibles para TRRC generan alarmas por encima de este valor ya que se producen flujos protrombóticos en el filtro.

\section{Cebado del circuito}

Inicialmente se prellena el circuito extracorpóreo con solución salina heparinizada con 5000 unidades por cada 1000cc, según la recomendación del fabricante. Posteriormente, se puede cebar con solución albuminada al $5 \%$ o glóbulos rojos de acuerdo con la necesidad clínica del paciente, en especial cuando el volumen sanguíneo de este, en el circuito extracorpóreo, sea mayor del 10\%. Lo anterior se realiza con el fin de prevenir hemodilución e hipotensión, y de limitar el riesgo asociado al desbalance electrolítico por hipercalcemia e hipocalcemia.

\section{Anticoagulación}

Una vez que la sangre entra en contacto con el circuito de circulación extracorpórea se activan los mecanismos de coagulación de la sangre, de manera que debe acompańarse de un método que evite que esto suceda. La anticoagulación permite que se optimice la vida funcional del circuito y del filtro, dado que la trombosis del filtro ocasiona un funcionamiento inadecuado y genera más costos para la terapia sustitutiva.

Dentro de las opciones de anticoagulación se encuentra, en primer lugar, la utilización de soluciones citratadas como método de anticoagulación regional. Ellas se recomiendan en pacientes sin contraindicaciones para el uso de citratos tales como enfermedad hepática, shock hepático, acidosis láctica y estados de hipoperfusión severa. Estas soluciones, utilizadas bajo protocolos estandarizados, no generan impacto en el estado de coagulación del paciente, no incrementan el riesgo de sangrado y permiten una larga vida del hemofiltro. Al usar soluciones citratadas existe riesgo de toxicidad por acumulación de citrato, además de hipocalcemia, hipernatremia y acidosis metabólica, por lo que se sugiere la monitorización del estado acido base, anión GAP y electrolitos. Adicionalmente, realizar el cálculo del índice de calcio, el cual permite realizar ajustes en la infusión de estas soluciones.

Otra opción para anticoagulación consiste en la administración de heparina no fraccionada a dosis de 20-40 UI/kg en bolo, seguida de una infusión de 5-15 $\mathrm{UI} / \mathrm{kg} / \mathrm{h}$. Se debe tener como control un tiempo de tromboplastina parcial activado (TTPa) con intervalo 1.5 a 2 veces del valor normal (35-45 seg). También puede administrarse enoxaparina a dosis de carga de $0.15 \mathrm{mg} / \mathrm{kg}$ e infusión de $0.05 \mathrm{mg} / \mathrm{kg} / \mathrm{h}$, utilizando control de factor anti-Xa $0.25-0.35 \mathrm{UI} / \mathrm{ml}$ en los sitios donde esté disponible $(21,22)$.

\section{Soluciones terapéuticas de TRRC}

Se debe elegir el líquido de tratamiento en caso de ser necesario según la modalidad de TRRC que se utilizará. El propósito de las soluciones utilizadas para la TRRC es adaptarse adecuadamente a la modalidad elegida con la mayor seguridad, que permita un adecuado control metabólico. Por lo tanto, la selección de solución depende del tipo alteración electrolítica y el tipo de anticoagulación.

Las soluciones disponibles en Colombia y su composición se muestran en la Tabla 5. 
Tabla 5. Soluciones terapéuticas para TRRC disponibles en Colombia

\begin{tabular}{|c|c|c|c|c|c|c|}
\hline Plasma & $\begin{array}{l}\text { Soluciones } \\
\text { de susti- } \\
\text { tución, } \\
\text { reposición } \\
\text { o diálisis }\end{array}$ & & $\begin{array}{c}\text { Anticoa- } \\
\text { gulación } \\
\text { local } \\
\text { citrato }\end{array}$ & & & \\
\hline & & $\begin{array}{c}\text { Solución } \\
\text { potasio 4/ } \\
\text { Calcio 2.5 } \\
\text { meq/1 }\end{array}$ & $\begin{array}{c}\text { Solución } \\
\text { Potasio } \\
\text { 2/ Calcio } \\
\text { 0meq/L }\end{array}$ & $\begin{array}{c}\text { Solución } \\
\text { Potasio } \\
\text { 0/ calcio } \\
3.5\end{array}$ & $\begin{array}{l}\text { Solución } \\
\text { Citrato } \\
\text { diluido }\end{array}$ & $\begin{array}{l}\text { Solución } \\
\text { con bajo } \\
\text { Bicarbona- } \\
\text { to sin calcio }\end{array}$ \\
\hline $\begin{array}{l}\text { Citrato } \\
\mathrm{mMol} / \mathrm{L}\end{array}$ & & 0 & 0 & 0 & 18 & 0 \\
\hline $\begin{array}{l}\text { Potasio } \\
(\mathrm{meq} / \mathrm{l})\end{array}$ & $3.5-5.0$ & 4 & 2 & 0 & 0 & 4 \\
\hline $\begin{array}{l}\text { Calcio } \\
(\mathrm{meq} / \mathrm{l})\end{array}$ & $2.3-2.6$ & 2.5 & 0 & 3.5 & 0 & 0 \\
\hline $\begin{array}{l}\text { Magnesio } \\
\text { (meq/l) }\end{array}$ & $1.4-2.0$ & 1.5 & 1.0 & 1.0 & 0 & 0.75 \\
\hline $\begin{array}{l}\text { Sodio } \\
(\mathrm{meq} / \mathrm{l})\end{array}$ & $135-145$ & 140 & 140 & 140 & 149 & 140 \\
\hline $\begin{array}{l}\text { Cloro } \\
(\mathrm{meq} / \mathrm{l})\end{array}$ & $100-108$ & 113 & 108 & 109.5 & 86 & 120 \\
\hline $\begin{array}{l}\text { Bicarbonato } \\
\text { (meq/l) }\end{array}$ & $22-26$ & 32 & 32 & 32 & 0 & 22 \\
\hline $\begin{array}{l}\text { Lactato } \\
\text { (meq/l) }\end{array}$ & $0.5-2.2$ & 3 & 3 & 3 & 0 & 3 \\
\hline $\begin{array}{l}\text { Dextrosa } \\
(\mathrm{mg} / \mathrm{dl})\end{array}$ & $70-110$ & 110 & 110 & 0 & 0 & 110 \\
\hline $\begin{array}{l}\text { Osmolari- } \\
\text { dad } \\
(\mathrm{mOsm} / \mathrm{l})\end{array}$ & $280-296$ & 300 & 292 & 287 & 244.0 & 296.4 \\
\hline
\end{tabular}

Elaborado por: Pantoja G, Mondragón M, Lasso A y Lasso R.

\section{Dosis entregada}

La dosis es la cantidad entregada de TRRC para control de toxinas urémicas. La recomendación inicial se puede calcular según las siguientes fórmulas:

- Dosis = 20-60 ml/kg/hr (recomendada 20-25 ml/ kg/h) Recomendación KDIGO 2012

- Dosis $=2000-3000 \mathrm{ml} / 1.73 \mathrm{~m} 2 / \mathrm{h}$

- Dosis $=8000 \mathrm{ml} / 1.73 \mathrm{~m} 2 / \mathrm{h}$ en hiperamoniemia
En hemodiafiltración venovenosa continua la dosis es mínima de $2000 \mathrm{ml} / \mathrm{hr} / 1.73 \mathrm{~m} 2$ y podría ser dividida igualmente entre diálisis y solución de reemplazo $(50 \%$ y $50 \%$ ), las cuales serán modificables de manera dinámica según los objetivos de nuestro paciente (1-2,14).

Sin embargo, sea cual sea la prescripción de los flujos de tratamiento, a excepción del uso de citrato diluido, es importante que la fracción de filtración no exceda más del $25 \%$, ya que esto sugiere un riesgo mayor de coagulación del filtro. Se entiende fracción de filtración como el porcentaje del total de agua filtrada en la membrana del total de volumen acuoso 
que entra al filtro. Actualmente los sistemas modernos calculan automáticamente y presentan al operador dicho parámetro, siendo el flujo de sangre el principal factor que afecta su incremento, como altos valores de flujos sustitutivos. De no contar con dicho valor, es recomendable calcularlo así:

Fracción de filtración $=$ UF del paciente + FSPre + FSPos

Flujo plasmático+ FSPre

Flujo Plasmático $=($ Flujo de sangre programado $\mathrm{x} 60) \mathrm{x}$ (1-Hematocrito (\%))

Abreviaturas: UF, Ultrafiltrado; FSPre, Flujo de sustitución prefiltro; FSPos, Flujo sustitución posfiltro

\section{Membranas/filtros}

Los filtros son membranas semipermeables que se eligen dependiendo de la superficie corporal del paciente, la modalidad de TRRC elegida, así como de la máquina y tecnología disponible. Un amplio número de hemofiltros y membranas han sido desarrolladas para el uso de TRRC. Las características de las membranas que se deben tener en cuenta son su biocompatibilidad y permeabilidad, el grosor, el tamańo de los poros, la carga y las propiedades adsortivas, las cuales determinarán la capacidad de estas para remover solutos durante la terapia.

Cada membrana tiene características intrínsecas de permeabilidad a solutos y agua que permiten clasificarlas por su coeficiente de ultrafiltración o transferencia de masa, además de generar muy baja resistencia al paso de sangre.

Algunas membranas son específicas para eliminar factores inflamatorios, citoquinas y endotoxinas; los tipos de material sintético más utilizados en la construcción de estas son: polisulfona, poliamida, acrilonitrilos, polietersulfonas (1-3).

\section{Ultrafiltración}

Se deben considerar límites en la ultrafiltración, se calcula a $0.5-2 \mathrm{ml} / \mathrm{kg} / \mathrm{hr}$ o $1-3 \%$ del volumen sanguíneo/ hora. La extracción dependerá del estado hemodinámico y tamańo del paciente, además de la modalidad elegida. Se ajustará según la meta individualizada que se ha definido de ultrafiltrado, teniendo en cuenta las necesidades específicas de eliminación de fluidos, que idealmente corresponden a la pérdida de al menos el 5\% del peso por día cuando el objetivo es un balance hídrico negativo. No obstante, es posible desarrollar terapias con ultrafiltración cero o incluso alcanzar balances positivos de acuerdo a la hemodinamia del paciente.

\section{Monitorización}

Se recomienda solicitar estudios de laboratorio cada 6 a 8 horas en caso de que se requiera corroborar algún parámetro sérico que sea de alto riesgo o para evaluar el parámetro que se definió como objetivo del aclaramiento. Estos estudios pueden incluir perfil hemático, química sanguínea, electrolitos completos, gasometrías y análisis de la coagulación $(1,2)$.

\section{FINALIZACIÓN DE LA TRRC}

Una vez que las metas planteadas al inicio se han cumplido se considerará suspender la TRRC, proceso denominado reto renal, el cual se puede complementar con la utilización de diuréticos para valorar la producción de orina, y con la evaluación paraclínica de la depuración de azoados u otras moléculas que sean de interés de aclaramiento (23).

Los criterios para el retiro de la terapia de reemplazo renal continua son:

- Estabilidad hemodinámica.

- Balance hídrico que permita acumular ligeramente fluido sin aumentar el riesgo de edema pulmonar.

- Electrolitos y estado ácido-básico normal.

- Volumen urinario adecuado según la edad.

- Transición a hemodiálisis intermitente o diálisis peritoneal.

Si el paciente se encuentra estable, pero aún requiere sesiones de hemodiálisis para equilibrio hídrico o de solutos, se recomienda cambiar a terapia de hemodiálisis intermitente, sobre todo para disminuir costos. 


\section{CONCLUSIONES}

La LRA es una entidad muy frecuente observada en pacientes críticamente enfermos; su presencia se relaciona con un aumento de la morbimortalidad.

La TRRC es una terapia compleja con múltiples ventajas sobre los procedimientos convencionales de diálisis. Su éxito depende de una adecuada prescripción, la cual se logra mediante el conocimiento de los conceptos básicos, estrategias y recursos disponibles. Realizamos esta revisión para entregar una herramienta práctica que facilite la prescripción de TRRC en pacientes pediátricos críticamente enfermos.

\section{CONTRIBUCIÓN DE LOS AUTORES}

OCPG: Planificación del estudio, revisión de la literatura, redacción del manuscrito.

MVMG: Planificación del estudio, revisión de la literatura, redacción del manuscrito.

ACLF: Planificación del estudio, revisión de la literatura, redacción del manuscrito.

RELP: Planificación del estudio, revisión de la literatura, redacción del manuscrito.

\section{CONFLICTOS DE INTERÉS}

Los autores declaramos que no existe conflicto de interés para la publicación del presente artículo.

\section{FUENTES DE FINANCIAMIENTO}

Ninguna.

\section{REFERENCIAS}

1. Sosa-Medellín MÁ, Luviano-García JA. Continuous renal replacement therapy. Concepts, indications and basic aspects of its program. Med Interna Mex. 2018; 34(2):288-98. https://doi.org/10.24245/ mim.v34i2.1652
2. Resendez T, Roque J. Manual De La Sociedad Latinoamericana De Cuidados Intensivos Pediatricos. 2016; (3):1-16.

3. Fernández S, Santiago MJ, González R, et al. Hemodynamic impact of the connection to continuous renal replacement therapy in critically ill children. Pediatr Nephrol. 2019; 34(1):163-8. https://doi.org/10.1007/s00467-018-4047-7

4. Beltramo F, DiCarlo J, Gruber JB, et al. Renal Replacement Therapy Modalities in Critically Ill Children. Pediatr Crit Care Med. 2019; 20(1):1-9. https://doi.org/10.1097/PCC.0000000000001754

5. Kaddourah A, Basu RK, Bagshaw SM, Goldstein SL. Epidemiology of Acute Kidney Injury in Critically Ill Children and Young Adults. N Engl J Med. 2017; 376(1):11-20. https://doi.org/10.1056/ NEJMoa1611391

6. Jetton JG, Boohaker LJ, Sethi SK, Wazir S, Rohatgi $S$, Soranno DE, et al. Incidence and outcomes of neonatal acute kidney injury (AWAKEN): a multicentre, multinational, observational cohort study. Lancet Child Adolesc Heal. 2017; 1(3):184-94. https://doi.org/10.1016/S2352-4642(17)30069-X

7. Chawla LS, Bellomo R, Bihorac A, Goldstein SL, Siew ED, Bagshaw SM, et al. Acute kidney disease and renal recovery: Consensus report of the Acute Disease Quality Initiative (ADQI) 16 Workgroup. Nat Rev Nephrol. 2017; 13(4):241-57. https://doi. org/10.1038/nrneph.2017.2

8. Andreoli SP. Acute kidney injury in children. Pediatric Nephrology. 2009; 24(2):253-63. https:// doi.org/10.1007/s00467-008-1074-9

9. Restrepo JM, Mondragon M V., Forero-Delgadillo JM, Lasso RE, Zemanate E, Bravo Y, et al. Acute renal failure in children. Multicenter prospective cohort study in medium-complexity intensive care units from the Colombian southeast. PLoS One. 2020; 15(8 August):1-11. https://doi.org/10.1371/ journal.pone.0235976

10. Restrepo de Rovetto C, Mora JA, Alexandre Cardona S, Marmolejo AF, Paz JF, de Castano I. Acute kidney injury applying pRifle scale in Children of Hospital Universitario del Valle in Cali, Colombia: clinical features, management and evolution. Colombia médica. 2012; 43(3):200-5.

11. Kellum JA, Lameire N, Aspelin P, Barsoum RS, Burdmann EA, Goldstein SL et al. Kidney disease: Improving global outcomes (KDIGO) acute kidney injury work group. KDIGO clinical practice guideline 
for acute kidney injury. Kidney International Supplements. 2012; 2(1):1-138. https://doi. org/10.1038/kisup.2012.1

12. Cabral FC, Garcia PCR, Mattiello R, Dresser D, Fiori HH, Korb C, et al. Influence of acute kidney injury defined by the pediatric risk, injury, failure, loss, end-stage renal disease score on the clinical course of PICU patients. Pediatr Crit Care Med. 2015; 16(8): e275-82. https://doi.org/10.1097/ PCC.0000000000000516

13. Hames DL, Ferguson MA, Kaza AK, Rajagopal S, Thiagarajan RR, Teele SA, et al. Renal replacement therapy in the pediatric cardiac intensive care unit. J Thorac Cardiovasc Surg. 2019; 158(5):1446-55. https://doi.org/10.1016/j.jtcvs.2019.06.061

14. Symons JM. Renal Replacement Therapy for the Critically Ill Infant. Third Edition. Critical Care Nephrology: Third Edition. 2017. P. 1230-1235.

15. Sethi SK, Mittal A, Nair N, Bagga A, Iyenger A, Ali U, et al. Pediatric Continuous Renal Replacement Therapy (PCRRT) expert committee recommendation on prescribing prolonged intermittent renal replacement therapy (PIRRT) in critically ill children. Hemodial Int. 2020; 24(2):237-51. https://doi.org/10.1111/hdi.12821

16. Symons JM, Chua AN, Somers MJG, Baum MA, Bunchman TE, Benfield MR, et al. Demographic characteristics of pediatric continuous renal replacement therapy: A report of the prospective pediatric continuous renal replacement therapy registry. Clin J Am Soc Nephrol. 2007; 2(4):732-8. https://doi.org/10.2215/CJN.03200906

17. Sutherland SM, Zappitelli M, Alexander SR, Chua AN, Brophy PD, Bunchman TE, et al. Fluid Overload and Mortality in Children Receiving Continuous Renal Replacement Therapy: The Prospective Pediatric Continuous Renal Replacement Therapy Registry. Am J Kidney Dis. 2010; 55(2):316-25. https://doi. org/10.1053/j.ajkd.2009.10.048

18. Selewski DT, Cornell TT, Blatt NB, Han YY, Mottes T, Kommareddi M, et al. Fluid overload and fluid removal in pediatric patients on extracorporeal membrane oxygenation requiring continuous renal replacement therapy. Crit Care Med. 2012; 40(9):2694-9. https://doi.org/10.1097/ CCM.0b013e318258ff01

19. Chawla L, Davison D, Brasha-Mitchell E, et al. Development and standardization of a furosemide stress test to predict the severity of acute kidney injury. Crit Care, 2013; 17(5): R207. https://doi. org/10.1186/cc13015
20. Hackbarth R, Bunchman TE, Chua AN, Somers MJ, Baum MA, Symons JM, et al. The effect of vascular access location and size on circuit survival in pediatric continuous renal replacement therapy: A report from the PPCRRT registry. Int $\mathrm{J}$ Artif Organs. 2007; 30(12):1116-21. https://doi. org/10.1177/039139880703001212

21. Raymakers-Janssen PAMA, Lilien M, van Kessel IA, Veldhoen ES, Wösten-van Asperen RM, van Gestel JPJ. Citrate versus heparin anticoagulation in continuous renal replacement therapy in small children. Pediatr Nephrol. 2017; 32(10):1971-8. https://doi.org/10.1007/s00467-017-3694-4

22. Brophy PD, Somers MJG, Baum MA, Symons JM, McAfee N, Fortenberry JD, et al. Multi-centre evaluation of anticoagulation in patients receiving continuous renal replacement therapy (CRRT). Nephrol Dial Transplant. 2005; 20(7):1416-21. https://doi.org/10.1093/ndt/gfh817

23. Uchino S, Bellomo R, Morimatsu H, Morgera S, Schetz M, Tan I, Bouman C, Macedo E, Gibney N, Tolwani A, Straaten HO, Ronco C, Kellum JA. Discontinuation of continuous renal replacement therapy: a post hoc analysis of a prospective multicenter observational study. Crit Care Med. 2009; 37(9):2576-82. https:// doi.org/10.1097/CCM.0b013e3181a38241 